\title{
Etude ethnobotanique sur Euphorbia heterophylla en Côte d'Ivoire
}

\author{
Joël Aholié DINGUI ${ }^{*}$, Casimir Yao BROU ${ }^{1}$, David N'goran KOUAKOU ${ }^{1}$ et \\ Noël Guédé ZIRIHI² \\ ${ }^{1}$ Institut National Polytechnique Félix HOUPHOUET BOIGNY (INPHB), Ecole Supérieure d'Agronomie \\ (ESA), BP 1313 Yamoussoukro, Côte d'Ivoire. \\ ${ }^{2}$ Université Félix HOUPHOUET BOIGNY, UFR Biosciences de COCODY, Abidjan, 22 BP 582 Abidjan 22, \\ Cote d'Ivoire. \\ *Auteur correspondant; E-mail: dinguijoel57@gmail.com, Tel : (+225) 0707497612
}

\begin{tabular}{lll}
\hline Received: 11-08-2021 & Accepted: 23-12-2021 & Published: 31-12-2021 \\
\hline
\end{tabular}

\section{RESUME}

En vue de mieux appréhender le niveau de connaissance de la population ivoirienne sur l'espèce Euphorbia heterophylla et d'enrichir la pharmacopée ivoirienne, une étude ethnobotanique a été réalisée. Elle s'est faite à partir d'une prospection au cours de laquelle des échantillons de plantes ont été prélevés dans les cinq zones géographiques de la Côte d'Ivoire, suivie d'une enquête dans certaines localités. La prospection a permis de ressortir 3 morphotypes caractérisés par 3 formes de tige : unique, peu ramifiée et très ramifiée ainsi que 3 formes de feuilles : ovale, violon et lancéolée. Sur le plan culturel, l'étude a révélé que $75 \%$ des personnes enquêtées connaissent la plante, 55\% la considèrent comme mauvaise herbe, 13,39\% estiment qu'elle sert à prodiguer des soins de santé aux humains. L'organe le plus utilisé est la feuille pour les soins à $100 \%$. Le mode de préparation le plus utilisé est le broyage de feuilles fraîches ou l'expression. Les modes d'applications sont surtout cutanés (90\%). Elle est fréquemment utilisée pour le traitement du paludisme, soit 33\% des cas. Les autres soins prodigués allaient contre les maladies infantiles dont la fièvre (11\%) et d'autres maux. Ces résultats peuvent servir de base de recherches en phytochimie et en pharmacologie.

(C) 2021 International Formulae Group. All rights reserved.

Mots clés : Euphorbia heterophylla, morphotypes, utilisation, Côte d'Ivoire.

\section{Ethnobotanical study on Euphorbia heterophylla in Côte d'Ivoire}

\begin{abstract}
In order to better understand the level of knowledge of the Ivorian population on the species Euphorbia heterophylla and to enrich the Ivorian pharmacopoeia, an ethnobotanical study was carried out. It was based on a survey during which samples of plants were taken in the five geographical areas of Côte d'Ivoire, followed by a survey in some localities. The survey allowed us to identify 3 morphotypes characterized by 3 stem shapes: single, little branched and very branched as well as 3 leaf shapes: oval, violin and lanceolate. On the cultural level, the study revealed that $75 \%$ of the people surveyed know the plant, $55 \%$ consider it as a weed, $13.39 \%$ believe that it is used to provide health care to humans. The most used organ is the leaf for health care at $100 \%$.
\end{abstract}


The most used method of preparation is crushing fresh leaves or expression. The modes of application are mainly cutaneous (90\%). It is frequently used for the treatment of malaria, 33\% of cases. The other treatments were for children's diseases, including fever $(11 \%)$ and other ailments. These results can serve as a basis for research in phytochemistry and pharmacology.

(C) 2021 International Formulae Group. All rights reserved.

Keywords: Euphorbia heterophylla, morphotypes, use, Côte d'Ivoire.

\section{INTRODUCTION}

Les bienfaits des plantes que nous offre la nature ont été découverts de façon progressive par les hommes depuis des siècles. Malheureusement, la transmission sans conservation écrite de ces connaissances a engendré quelquefois des pertes d'informations précieuses, surtout dans les pays sous-développés et singulièrement en Afrique. Avec l'évolution due aux progrès technologiques et philosophies sociales sur tous les continents, le recours aux plantes semble salvateur pour le bien être des humains, d'où la nécessité de la préservation de la biodiversité, objet de combat quotidien des scientifiques. Ainsi, les plantes font l'objet d'attraction de nombreux chercheurs à l'échelle mondiale entre autres, les ethnobotanistes qui ont prouvé l'efficacité de la phytothérapie ainsi que l'incontestabilité de leurs bienfaits (Bene et al., 2016). En Afrique, l'intérêt pour les plantes s'est agrandi du fait de la paupérisation grandissante qui ne permet pas à une frange importante de la population d'avoir accès aux produits pharmaceutiques conventionnels. Selon l'OMS (2002), environ $80 \%$ des populations africaines ont recours à la médecine traditionnelle pour satisfaire leurs besoins en soins de santé primaires. En Côte d'Ivoire, depuis quelques décennies plusieurs publications de travaux de plusieurs auteurs ayant trait aux bienfaits des plantes surtout médicinales ont été faites. Il s'agit entre autres de Koné et al (2002), Ouattara (2006), N'guessan (2008), Yapi (2013) cités par Bene (2016).

L'espèce Euphorbia hirta par exemple est traditionnellement utilisée comme antidote (thérapie anti-venin) pour les morsures de serpent et aliments selon Kadiyala et al. (2015). La consommation d'œufs de poules pondeuses (ISA Warren) ayant reçu $80 \%$ de régime contrôlé, $5 \%$ de complément minéral vitaminé et $15 \%$ de graines de Euphorbia heterophylla conduit à une baisse significative des proportions d'acides gras saturés, mono insaturés, polyinsaturés n-3, et permettrait d'améliorer la prévention des maladies cardiovasculaires (Kouakou et al., 2009). Falodun et al. (2006) ainsi que Oyedum (2017) ont respectivement montré les propriétés anti inflammatoires et les propriétés antibactériennes de Euphorbia heterophylla. Selon James et al. (2014) le genre Euphorbia présente différents aspects botaniques. Ces différentes études n'ont pas scruté les potentialités que renferme cette plante pour la Côte d'Ivoire sur le plan médicinal, nutritionnel et économique. Cette étude a donc pour objectif de relever ces potentialités et d'enrichir la pharmacopée ivoirienne.

\section{MATERIEL ET METHODES}

\section{Milieu de l'étude}

L'étude s'est déroulée en Afrique occidentale, principalement en Côte d'Ivoire, pays d'une superficie de $322462 \mathrm{Km}^{2}$, dans les différentes zones géographiques regroupées en zones écologiques, à savoir : le Nord (zone Subsoudanienne), l'Ouest (zone Pré forestière), le Centre (à cheval sur les zones Moyenne Côte d'Ivoire et Basse Côte d'Ivoire, l'Est (Moyenne Côte d'Ivoire), et le Sud (Basse Côte d'Ivoire forestière). Les coordonnées géographiques de ces différentes zones sont $9^{\circ} 16^{\prime}-10^{\circ} 29 \quad$ latitude, $5^{\circ} 12^{\prime}-7^{\circ} 34^{\prime}$ longitude (Nord) ; $5^{\circ} 32^{\prime}-8^{\circ} 17^{\prime}$ latitude, $7^{\circ} 21^{\prime}$ $7^{\circ} 41^{\prime}$ longitude (Ouest); 6 $34^{\prime}-7^{\circ} 45^{\prime}$ latitude, 
$4^{\circ} 59^{\prime}-6^{\circ} 04^{\prime}$ longitude (Centre) ; 6 $6^{\circ} 39^{\prime}-7^{\circ} 08^{\prime}$ latitude, $4^{\circ} 50^{\prime}-2^{\circ} 47^{\prime}$ longitude (Est); $4^{\circ} 25^{\prime}$ $6^{\circ}$ latitude, $7^{\circ} 22^{\prime}-2^{\circ} 47^{\prime}$ longitude (Sud). Les localités enquêtées sont celles où la plante existe et est connue par au moins une frange de la population surtout rurale ou péri-urbaine. Il s'agit, de Korhogo et Odienné (au nord: Régions du Poro et du Kabadougou); de Bangolo (à l'Ouest: Région du Guémon); de Yamoussoukro et Zuenoula (au Centre: Régions du Bélier et de la Marahoué); d'Abengourou et Niable (à l'Est: Région de l'Indénié-Djuablin); et de Soubré et Bonoua (au Sud: Régions de la Nawa et du SudComoé).

\section{Matériel Végétal}

Il est représenté par les différents morphotypes d'Euphorbia heterophylla trouvées dans les localités (plantes à tiges unique, peu ramifiée ou très ramifiée avec des formes de feuilles ovale ou elliptique (a), violon (b), lancéolée (c), à bordures dentée ou pas (Figure 1).

\section{Matériel technique}

Cette étude a nécessité un matériel classique pour le recueil des informations et la récolte des échantillons de plantes. Il s'agit principalement de fiches d'enquêtes, de sachets en plastique (polyéthylène blanc et noir) de dimensions diverses (grande et petite), d'appareil photographique et de GPS.

\section{Méthodes d'étude Sites visités}

La Côte d'Ivoire étant composée de Zones géographiques comprenant des Régions divisées en Départements, eux-mêmes constitués de villages, les cinq zones géographiques ont été visitées. Au niveau de chaque zone, un village au moins a été choisi de façon aléatoire, là où lors de la première prospection, l'existence de la plante a été constatée. Il s'agit donc d'échantillons non probabilistes. En zone Nord, dans les régions du PORO et du KABADOUGOU, les
Départements (Korhogo et Odienné) et le village de Nammasselikaha ; à l'Ouest, Région du GUEMON, le département de Bangolo, les villages (Ponon, Biéou , Kahi, Ziondrou, Goénié, Béoua, Diapléan et Glodé) ; au Centre, Région de la MARAHOUE, le Département de Zuénoula, les villages (Scazra, Nénézra, Kanzra, Nincro, Gouhira, Gouéhizra,Kouablézra, Bonéfla, Zorofla et Srazra), Région du BELIER, Département de Yamoussoukro, village (Kami et Djahakro); Zone de l'Est, Région de l'Indenie-Djuablin , Départements d'Abengourou et de Niablé, villages (Yaokro et Akrabo); au Sud, Région de NAWA, Département de Soubré, villages (Gnity, Guéyo, Gabiadji et N'guessankro) et Région du SUD COMOE, Département de Grand-Bassam, village (Yaou) (Tableau 1 ). Les coordonnées géographiques satellitaires des Localités prospectées sont présentées dans le Tableau 2.

\section{Enquête}

Echantillonnage enquêtés : En moyenne 30 personnes ont été interrogées par Zone géographique. Le nombre total d'enquêtés est de 153 personnes.

Prélèvement de plants: Les échantillons de plants ont été prélevés dans chaque localité où s'est effectuée l'enquête, dans une jachère accessible et sur une terre ferme. Il s'agit de plants d'Euphorbia heterophylla sains, luxuriant, en croissance ou en pleine floraison ou encore portant des fruits et extirpé du sol avec ses racines. Ces plants sont présentés frais à l'enquêté.

Questionnaire : Les enquêtes ethnobotaniques ont été menées auprès des populations de chaque localité sans tenir compte de leurs activés. Elles ont été faîtes de façon aléatoire et les personnes disponibles ont répondu librement aux questions posées. Dans certains cas, il a été fait appel à un interlocuteur trouvé sur place et dont la compréhension et l'interprétation de la langue locale ont été testées pour la traduction. La plante était présentée pendant l'entretien. Les informations 
recueillies sur l'enquêté étaient : le nom, le sexe, l'âge, le niveau d'études, le groupe ethnique, la nationalité, l'activité. Les données concernant la plante et les usages qui en sont faits étaient : la connaissance de la plante, le nom local, l'utilité de la plante, l'aspect médical (les soins faits avec la plante, les organes utilisés, les modes de préparation et d'administration).

\section{Analyse statistique}

L'analyse des données a été réalisée de la façon suivante: dépouillement des fiches d'enquête, puis analyse statistique des résultats. En procédant ainsi, les réponses aux questionnaires ont été dépouillées, saisies et enregistrées à l'aide du tableur EXCELL 2010. Les données ont ensuite été traitées avec le logiciel informatique STISTICA 7.
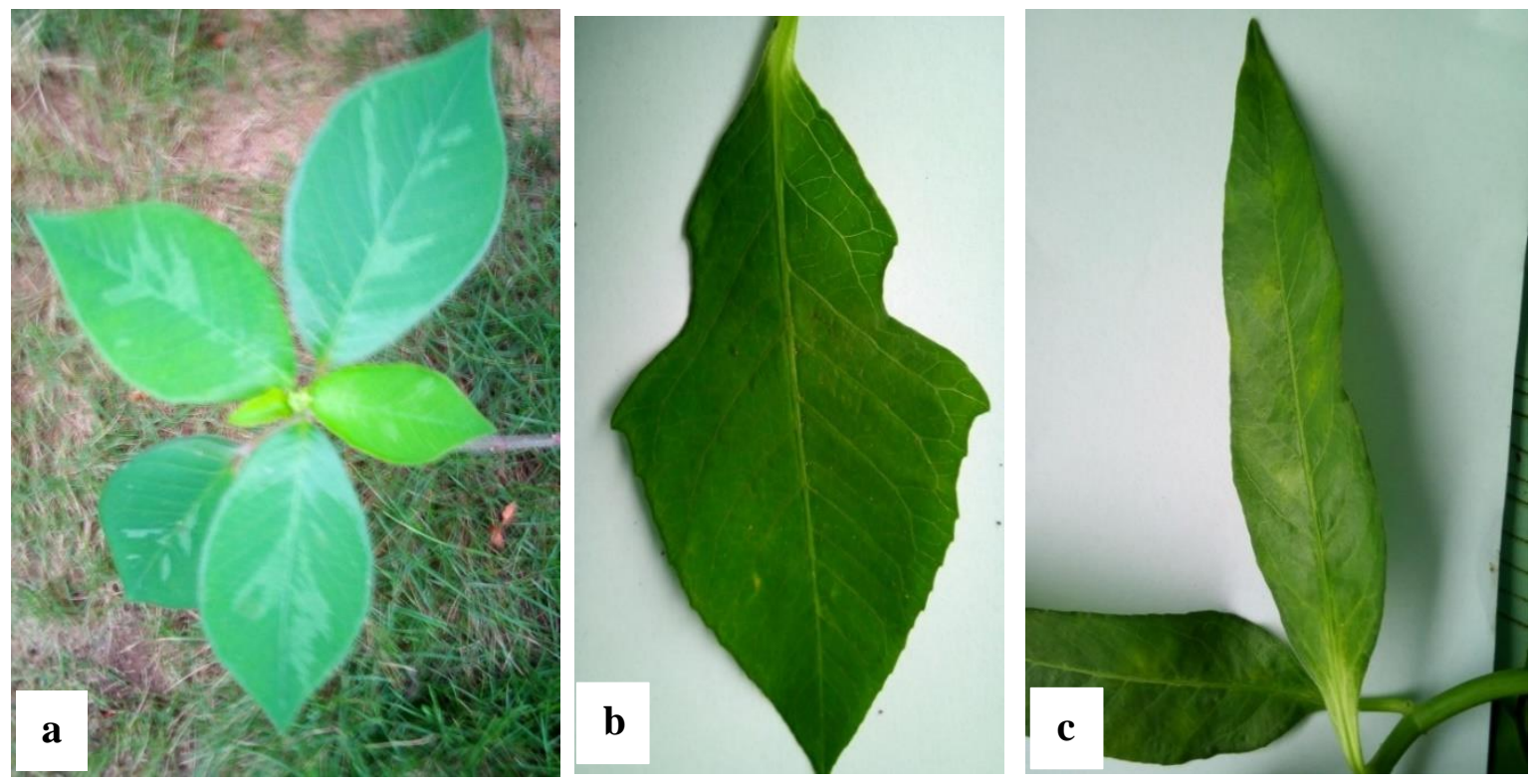

Figure 1: Les différentes formes de feuille d'Euphorbia heterophylla rencontrées.

Feuille ovale ou elliptique (a), Feuille violon (b), Feuille lancéolée (c)

Tableau 1: Coordonnées géographiques des Zones d'implantation d'Euphorbia heterophylla prospectées en Côte d'Ivoire.

\begin{tabular}{llll}
\hline Zone géographique & Zone écologique & Latitude & Longitude \\
\hline Nord & Subsoudanienne & $9^{\circ} 16^{\prime}-10^{\circ} 29$ & $5^{\circ} 12^{\prime}-7^{\circ} 34^{\prime}$ \\
\hline Ouest & Pré forestière & $6^{\circ} 32^{\prime}-8^{\circ} 17^{\prime}$ & $7^{\circ} 21-7^{\circ} 41^{\prime}$ \\
\hline Centre & Moyenne C.I et Bas-CI forestière & $6^{\circ} 34-7^{\circ} 45^{\prime}$ & $4^{\circ} 59^{\prime}-6^{\circ} 04$ \\
\hline Est & Moyenne Côte d'Ivoire & $6^{\circ} 39^{\prime}-7^{\circ} 08^{\prime}$ & $4^{\circ} 50-2^{\circ} 47^{\prime}$ \\
\hline Sud & Basse Côte d'Ivoire forestière & $4^{\circ} 25^{\prime}-6^{\circ}$ & $7^{\circ} 22^{\prime}-2^{\circ} 47^{\prime}$ \\
\hline
\end{tabular}


Tableau 2 : Coordonnées satellitaires des Localités prospectées en Côte d'Ivoire.

\begin{tabular}{|c|c|c|c|}
\hline NOM & Type & $\mathbf{X}$ & $\mathbf{Y}$ \\
\hline KANIASSO & Village & $-1871,1562$ & 1013968,068 \\
\hline Tieme & Village & 490353,259 & 1057763,479 \\
\hline Namasselikaha & Village & 197538,496 & 982512,4395 \\
\hline Tieme & Village & 76465,8606 & 901382,2693 \\
\hline Kpato & Village & 241904,834 & 811013,4487 \\
\hline Pouezra & Village & 144357,308 & 823019,5843 \\
\hline Nenezra 2 & Village & 143682,216 & 810400,2723 \\
\hline Bozi-II & Village & 210843,436 & 761152,0049 \\
\hline Ahounien Foutou & Village & 347879,404 & 749757,0293 \\
\hline Niable & Village & 194405,596 & 680880,028 \\
\hline Ponan & Village & 3806,4634 & 653671,8023 \\
\hline AboudeKouassikro & Village & 326889,49 & 652129,6986 \\
\hline Tieme & Village & 87377,0306 & 643886,3772 \\
\hline Touagui I & Village & 103385,005 & 610671,9936 \\
\hline Touagui II & Village & 105436,383 & 601469,8571 \\
\hline Menegbe & Village & 104223,088 & 565249,3182 \\
\hline SAMATIGUILA & Chef-lieu de Sous-Préfecture & $-917,2078$ & 1089191,182 \\
\hline TIEME & Chef-lieu de Sous-Préfecture & 25884,4654 & 1058120,515 \\
\hline ODIENNE & Chef-lieu de Région & $-1452,7657$ & 1053816,263 \\
\hline MINIGNAN & Chef-lieu de Sous-Préfecture & $-1005,0633$ & 1108749,91 \\
\hline TENGRELA & Chef-lieu de Département & 126737,423 & 1160171,114 \\
\hline BOUNDIALI & Chef-lieu de Département & 117723,634 & 1054548,515 \\
\hline SIRASSO & Chef-lieu de Sous-Préfecture & 159622,928 & 1026920,826 \\
\hline SINEMANTIALI & Chef-lieu de Sous-Préfecture & 237829,716 & 1060441,124 \\
\hline YAMOUSSOUKRO & District & 249256,314 & 755057,3057 \\
\hline BONOUA & Chef-lieu de Sous-Préfecture & 434162,715 & 582813,5671 \\
\hline
\end{tabular}




\section{RESULTATS}

La présence de l'espèce Euphorbia heterophylla a d'abord été observée lors des premières investigations dans les différentes régions où les enquêtes ont été menées. La population enquêtée était composée de $25 \%$ de sexe féminin et $75 \%$ de sexe masculin (Figure 2). La composition de la population interrogée révèle que les classes d'âge les plus représentées étaient principalement celles de 30-39 ans ; 40-49 et 50-59 ans (Figure 3).

Les données obtenues montrent que la population rurale ou périurbaine des zones enquêtées connait l'Euphorbia heterophylla. Cela confirme le fait que l'agriculture occupe la plus grande partie de la population active en Côte d'Ivoire. Ainsi, selon les résultats, la population de la zone nord est la plus représentée (91\%). Ensuite viennent les populations des localités du Centre (89\%) et de l'Est $(83 \%)$. Il en est moins pour ceux des localités de l'Ouest (60\%) et du Sud (38\%) (Figure 4).

La plupart des enquêtés considèrent la plante comme nouvelle ou alors la rangent du côté des mauvaises herbes. Certains au contraire estiment que c'est une plante prisée comme aliment par certains animaux comme les lapins, les cobayes, les moutons, les bovins ou les animaux sauvages. Une frange très restreinte de la population dispose d'un savoir sur les usages médicinaux de l'Euphorbia heterophylla (Figure 5).

Les populations du nord et de l'Est ont la même perception de la plante mais à des proportions différentes. Au nord, toute la population interrogée la reconnait comme mauvaise herbe et $16 \%$ savent aussi qu'elle est prisée par les animaux d'élevage comme aliment. A l'Est par contre $100 \%$ des personnes interrogées disent qu'elle est à la fois une mauvaise herbe et aliment des animaux. A l'Ouest, au Centre et au Sud, elle est perçue à la fois comme mauvaise herbe, aliment des animaux et plante médicinale dans les proportions suivantes :
- Mauvaise herbe: Ouest (47\%), Centre (100\%), Sud (58\%);

- Nourriture d'animaux : Ouest (47\%); Centre (93\%); Sud (13\%);

- $\quad$ Soins humains : Ouest $(7 \%)$; Centre (18\%) ; Sud (29\%).

\section{Soins humains}

Sur le plan médicinal, Euphorbia heterophylla est utilisée pour soigner plusieurs maladies ou prévenir certains risques (annihiler des effets de dangers ultérieurs). Ainsi ces maladies ou préventions étaient le paludisme (33\%); l'antidote contre les morsures de serpents $(11 \%)$; la facilitation d'accouchement $(11 \%)$; la teigne $(11 \%)$; les maux d'oreille $(6 \%)$; la gonococcie $(5 \%)$; les plaies de ventre $(5 \%)$; la sinusite $(6 \%)$; le corps chaud d'enfant (6\%) et les dartres (6\%) (Figure 6).

\section{Mode de préparation}

Les modes de préparation varient en fonction des affections ou soins préventifs à prodiguer. La macération, la décoction, l'infusion et le broyage des feuilles sont les principales techniques de préparation. Des enquêtes, il ressort que l'expression (37\%) et le broyage (36\%) étaient les principaux modes de préparation des recettes (Figure 7).

\section{Voies d'administration :}

Il ressort des investigations que la voie cutanée était la plus utilisée (55\%). Outre cette voie, la voie orale $(18 \%)$ et d'autres voies ( $9 \%)$ chacune ont été enregistrées. Il s'agit de la voie auditive, nasale et anale (Figure 8).

\section{Organe de plante utlisé :}

Les feuilles constituaient l'organe le plus utlisé dans toutes les recettes. Les recettes à base de feuilles uniquement représentent $88 \%$ des préparations (Figure 9 ).

En outre, il existait des recettes à base de la plante entière et d'autres à partir de la plante sans les racines, celles-ci représentent $6 \%$ chacune. 


\section{Noms locaux donnés à la plante}

Le nom donné à la plante est fonction du constat fait par l'auteur sur la plante et son environnement. En effet, $55 \%$ des noms traduisaient ses dégâts de mauvaise herbe et sa propagation rapide ; $20 \%$ des noms se réfèrent à la sève de la plante comparée au lait ; $5 \%$ font allusion au bruit d'explosion des graines à maturité ; $5 \%$ à son bon développement végétatif ; $5 \%$ à l'animal et $10 \%$ à des comportements humains. Les noms locaux pouvaient varier au sein d'un même groupe ethnique dans la même localité. Les groupes ethniques ayant donné des noms étaient au nombre de dix (10), et les noms se chiffraient à vingt (20) (Tableau 3). Les nationalités des auteurs de ces noms étaient à dominance ivoirienne.

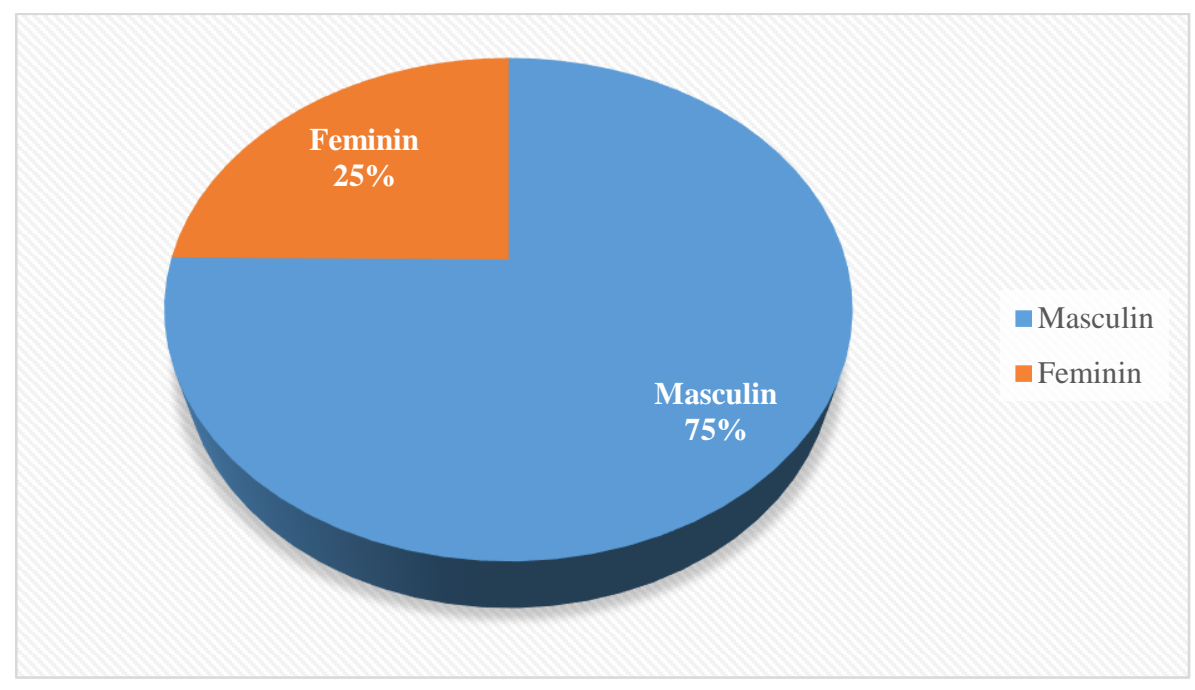

Figure 2 : Répartition de la population enquêtée selon le sexe.

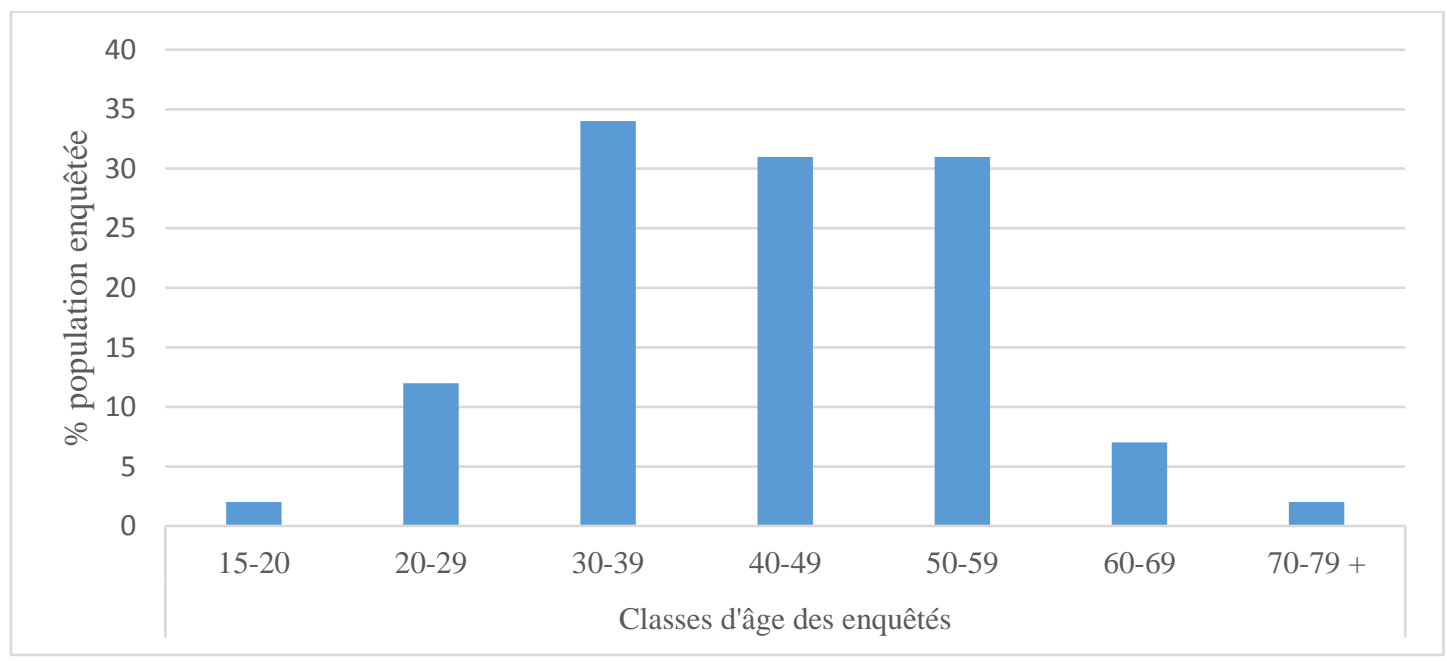

Figure 3 : Répartition de la population enquêtée selon les classes d'âge. 


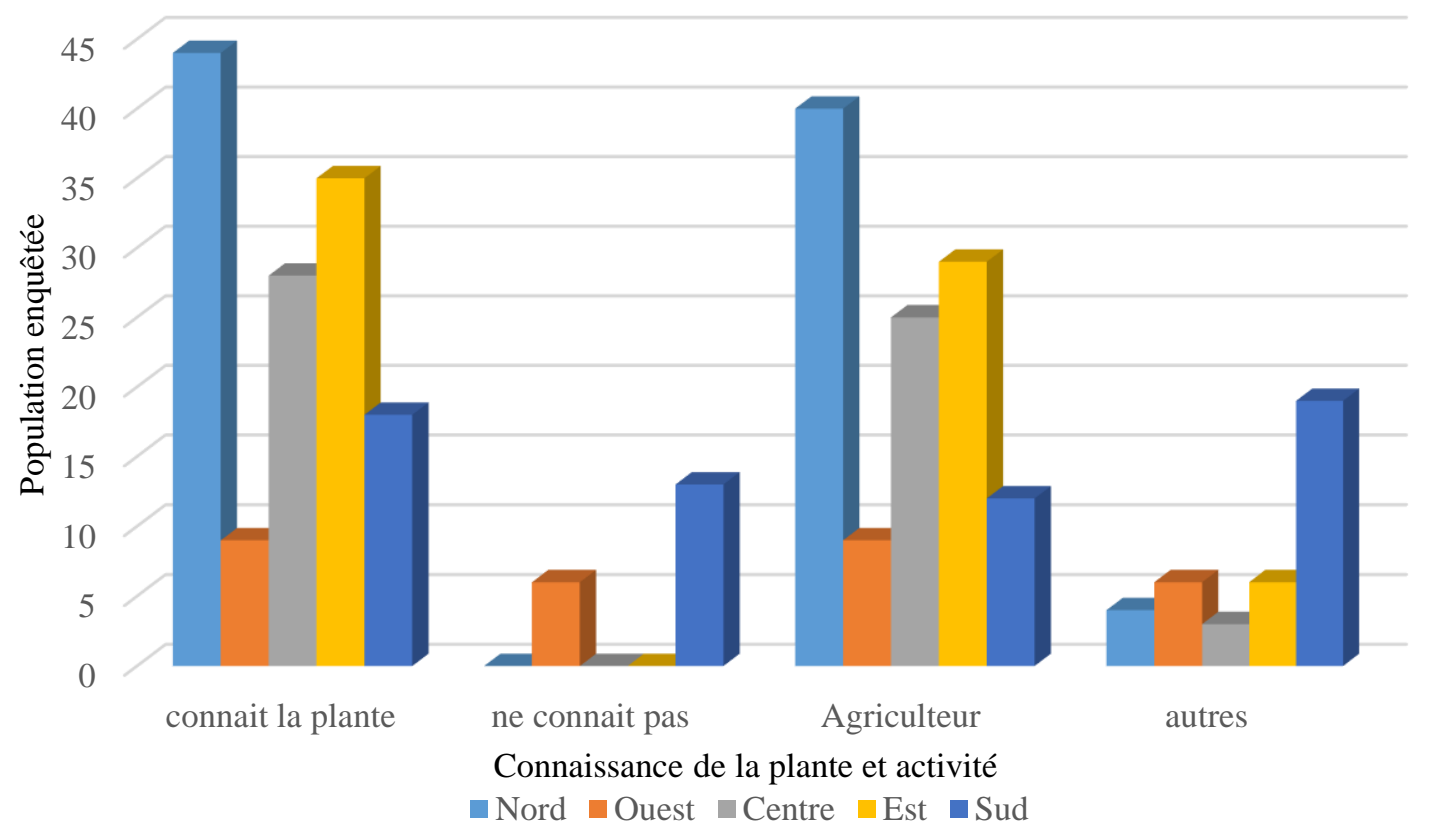

Figure 4 : Répartition de la population enquêtée selon la connaissance de la plante et l'activité.

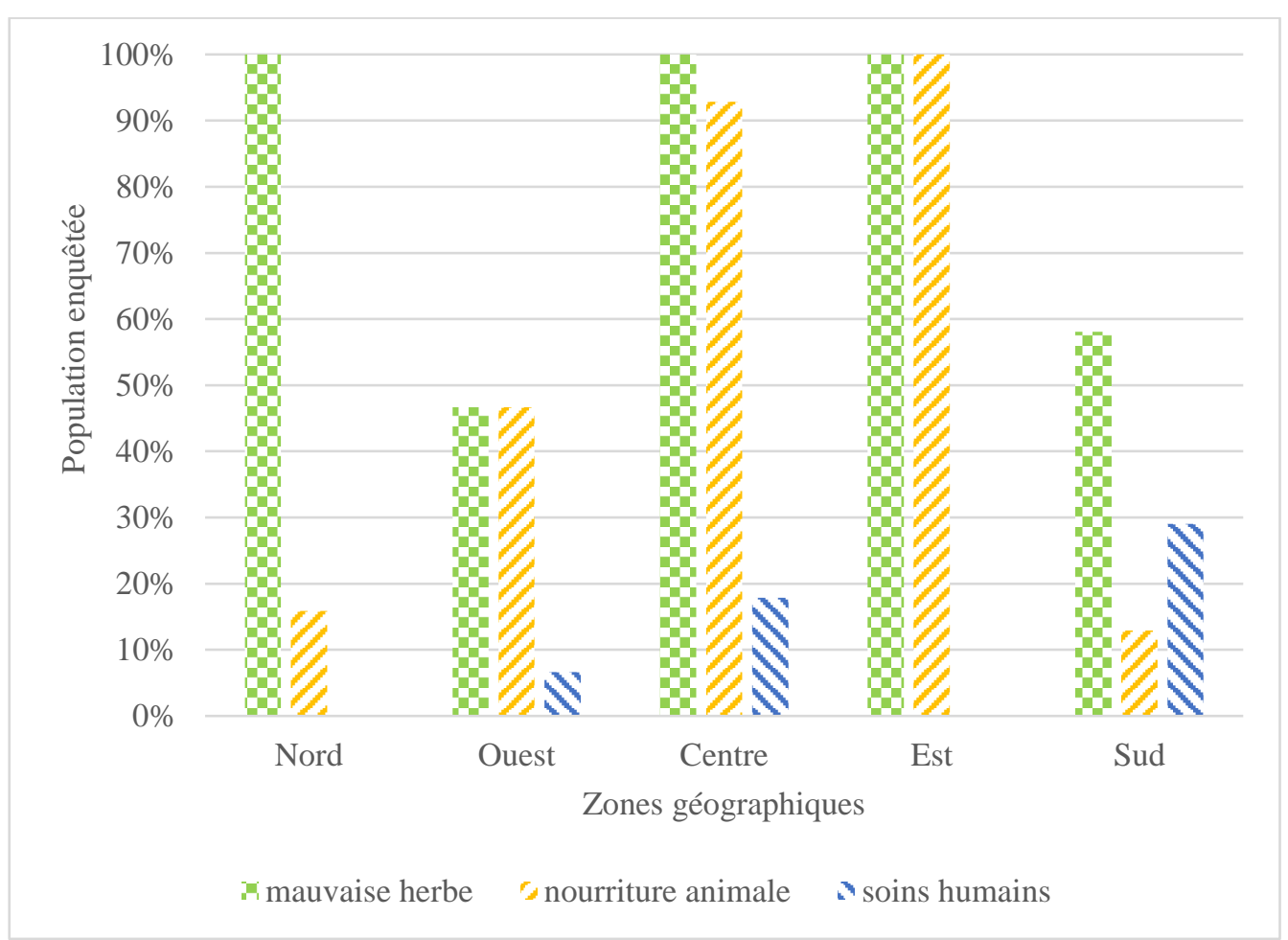

Figure 5 : Point de vue de la population enquêtée sur Euphorbia heterophylla. 


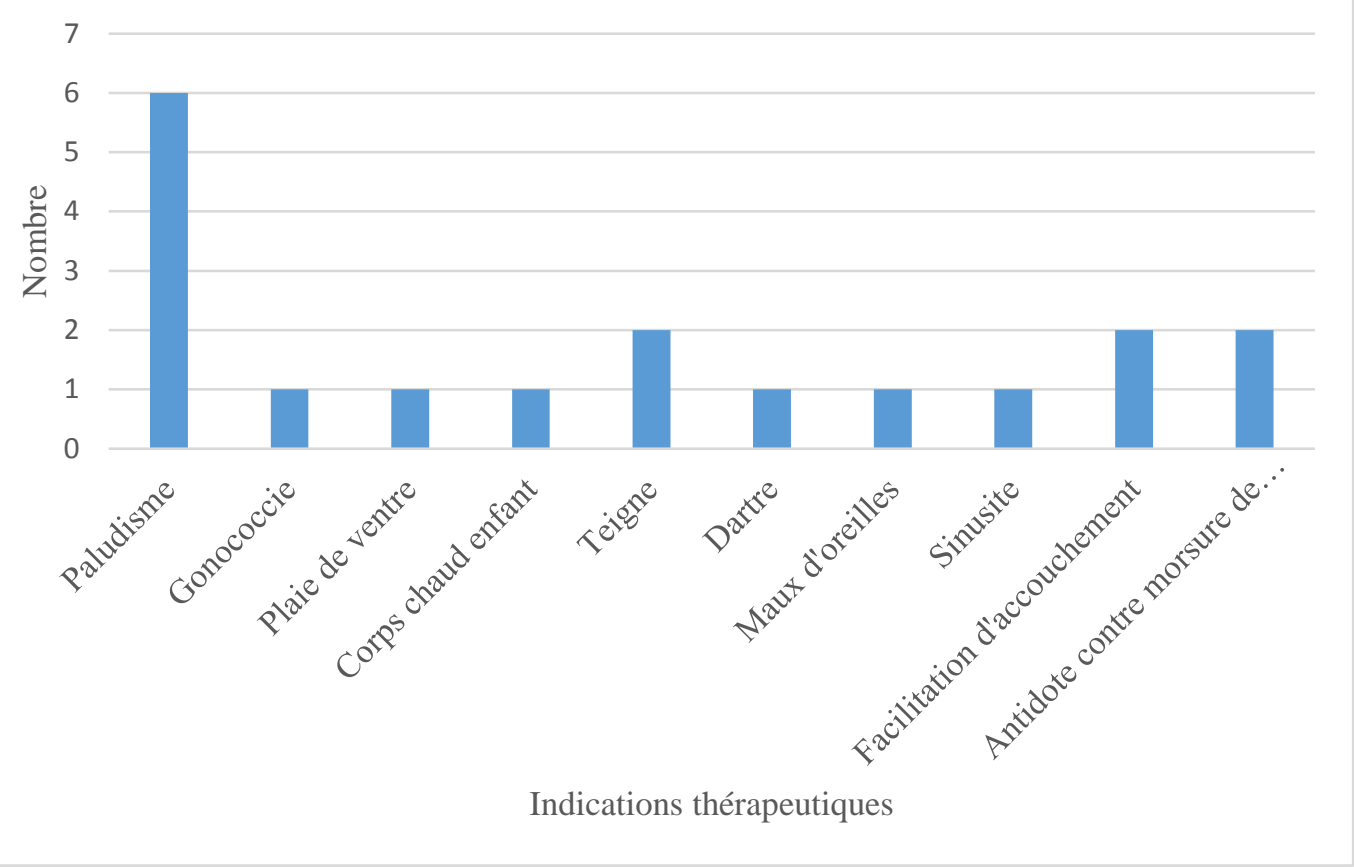

Figure 6 : Principales indications thérapeutiques.

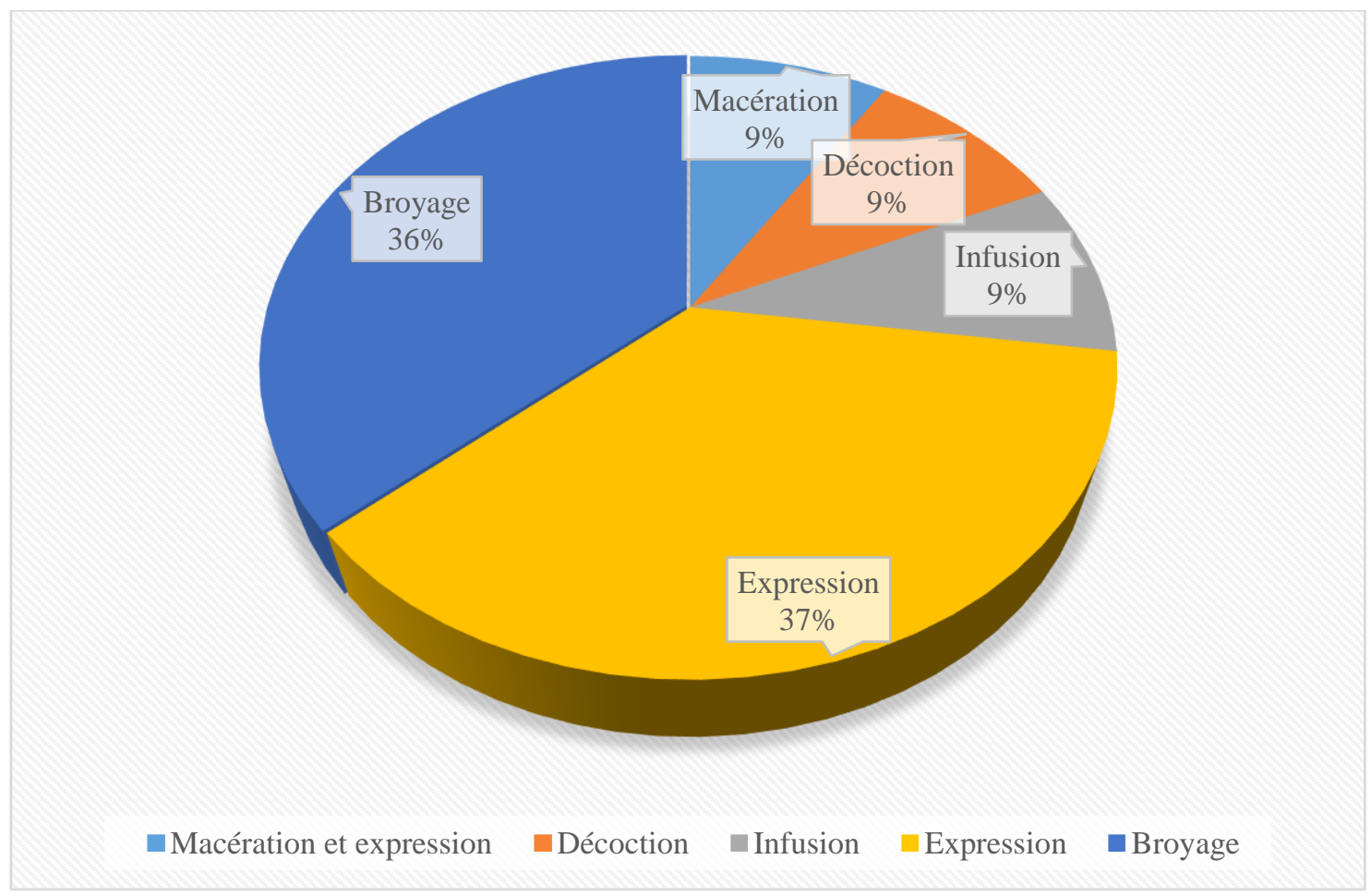

Figure 7 : Techniques de préparation des recettes. 


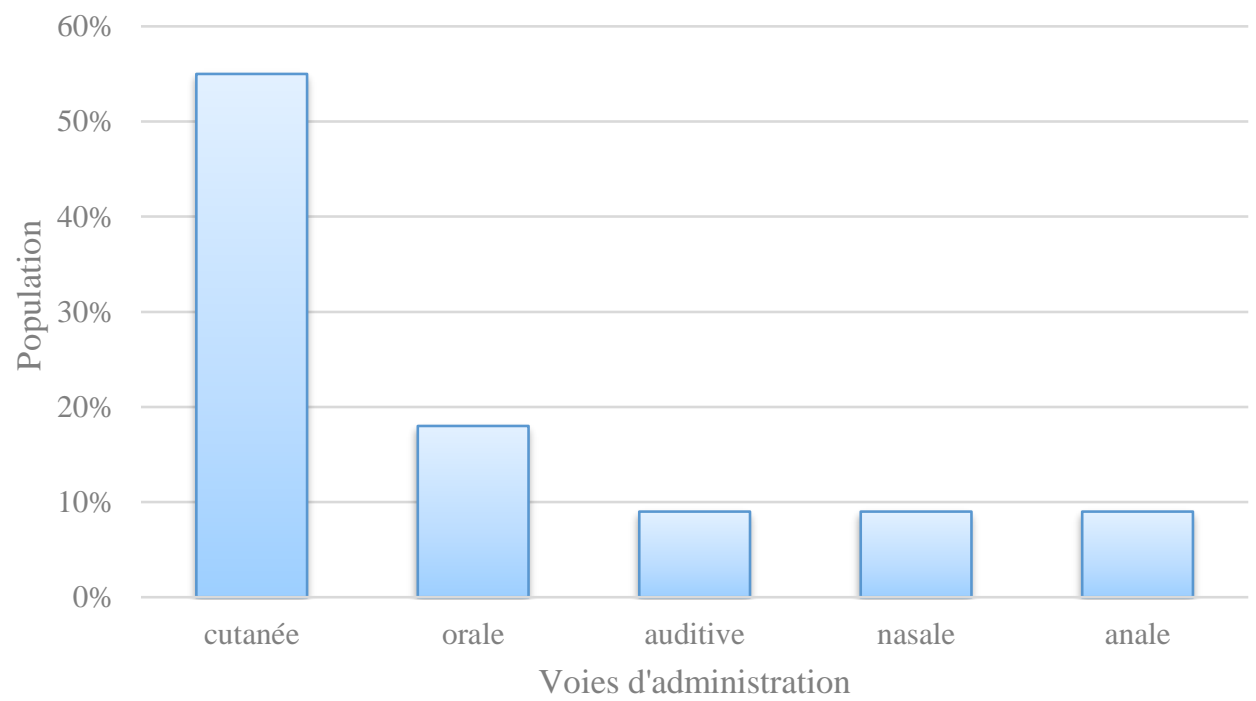

Figure 8 : Voies d'administration des phytomédicaments.

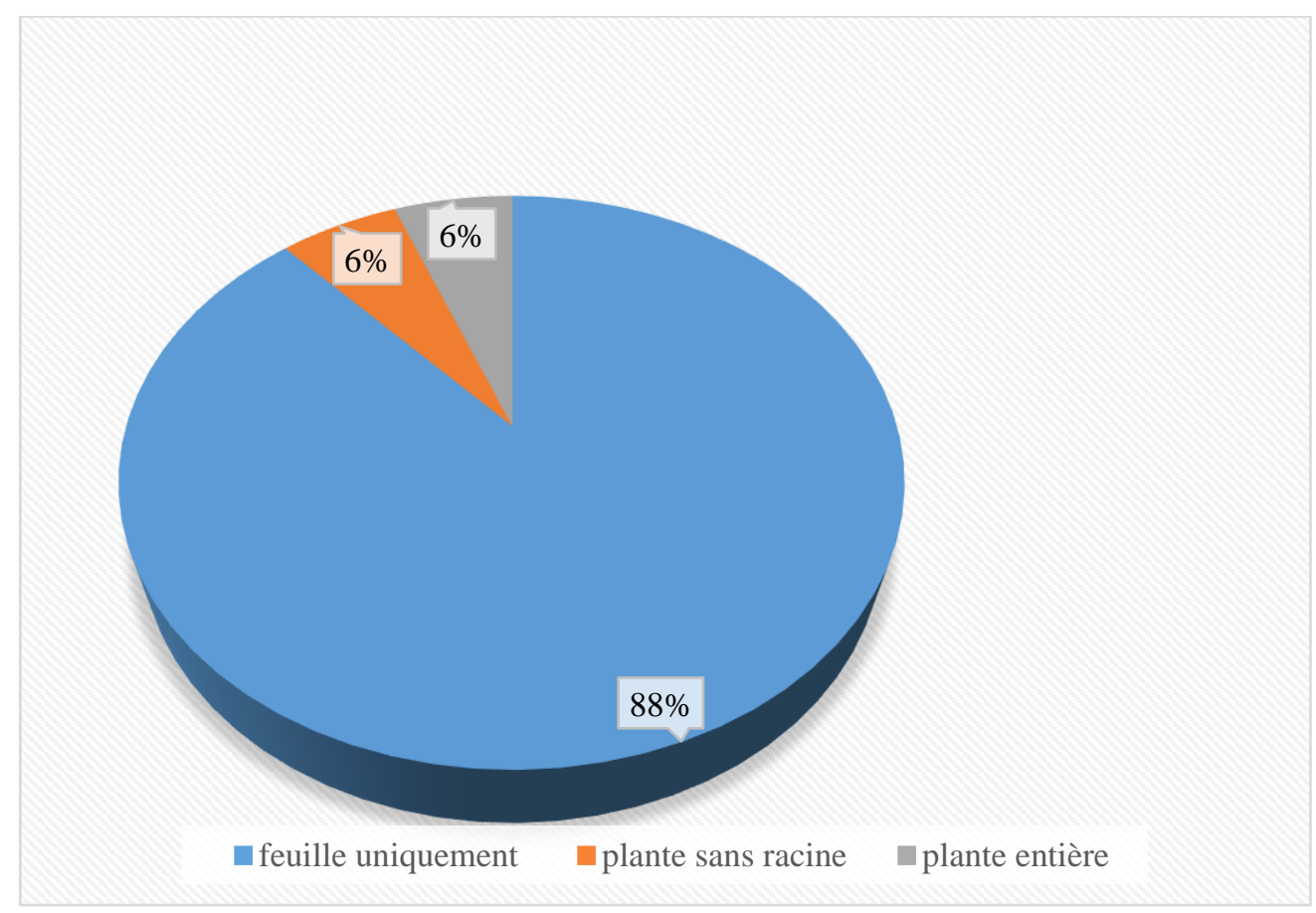

Figure 9: Organes utilisés de la plante. 
Tableau 3 : Noms locaux donnés à la plante.

\begin{tabular}{|c|c|c|c|c|}
\hline Pays d'origine & Localité & $\begin{array}{l}\text { groupe } \\
\text { ethnique }\end{array}$ & $\begin{array}{l}\text { nom } \\
\text { vernaculaire }\end{array}$ & signification française \\
\hline \multirow[t]{18}{*}{ Côte d'Ivoire } & Korhogo & & Katognimibi & $\begin{array}{l}\text { la plante s'installe, colonise le territoire } \\
\text { en tuant toutes les herbes }\end{array}$ \\
\hline & \multirow{3}{*}{ Kanzra } & \multirow{6}{*}{ sénoufo } & Tiafifara & $\begin{array}{l}\text { la plante s'installe, colonise le territoire } \\
\text { en tuant toutes les herbes }\end{array}$ \\
\hline & & & aboulaye & ça pousse trop, très dense \\
\hline & & & sewrenan & ça pousse trop \\
\hline & \multirow{4}{*}{$\begin{array}{l}\text { Korhogo } \\
\text { Namasselikaha } \\
\text { Kanzra }\end{array}$} & & donitcha & $\begin{array}{l}\text { si tu n'es pas courageux tu abandonnes } \\
\text { ton champ }\end{array}$ \\
\hline & & & donikan & Herbe méchante \\
\hline & & & Flatchal & Femme Peul \\
\hline & & tagbana & gnafie & ça pousse vite \\
\hline & Nénézra & malinké & Amanandjonan & $\begin{array}{l}\text { si tu n'étais pas venu vite, la nettoyer } \\
\text { serait difficile }\end{array}$ \\
\hline & $\begin{array}{l}\text { Skazra } \\
\text { (Zuénoula) }\end{array}$ & \multirow[t]{3}{*}{ gouro } & yadalai edrelo & Tu es difficile à nettoyer \\
\hline & Kanzra & & $\begin{array}{l}\text { Fehissia/ } \\
\text { Flassia }\end{array}$ & Elle prend tout le champ \\
\hline & Zorofla & & gnibo & ça donne du jus \\
\hline & Diaplean & guéré & lait sihi & plante à lait \\
\hline & Yamoussoukro & \multirow[t]{2}{*}{ Baoulé } & têtêkpokpo & $\begin{array}{l}\text { bruit de l'éclatement ou explosion des } \\
\text { fruits à maturité }\end{array}$ \\
\hline & Kanzra & & n'djissiwrolo & fragile à la sécheresse \\
\hline & $\begin{array}{l}\text { Yaou } \\
\text { (Bonoua) }\end{array}$ & Abouré & ploplo & qui se développe bien \\
\hline & $\begin{array}{l}\text { Yaokro } \\
\text { (Abengourou) }\end{array}$ & \multirow[t]{2}{*}{ Agni } & Mossi adja & pied de la femme mossi \\
\hline & Niablé & & acoq dodo & crête de coq \\
\hline Guinée & Kanzra & Guinéen & Baka & Ne meurt pas vite \\
\hline \multirow[t]{2}{*}{ Burkina Faso } & Doumbiakro & \multirow[t]{2}{*}{ moré } & mossingbri & herbe de lait \\
\hline & Kanzra & & wanbissin & herbe de pigeon (aime la sève) \\
\hline
\end{tabular}




\section{DISCUSSION}

La plupart de la population de la zone nord sont des agriculteurs et ils connaissent les dégâts que cause l'espèce Euphorbia heterophylla à leurs cultures. Ce résultat corrobore celui d'Ipou et al. (2005) qui révèlent que cette espèce végétale envahit les champs de cotonniers au nord de la Côte d'Ivoire. La forte proportion au niveau de cette zone en ce qui concerne la connaissance de la plante est liée aux cultures pratiquées. En effet, ces cultures dominantes, à savoir le coton, principale culture de rente et les céréales tels le maïs, le riz, le fonio et le mil destinées à la consommation, laissent généralement passer dans les interlignes de culture plus de lumière, facteur écologique favorable au développement de l'espèce Euphorbia heterophylla, plante héliophile, cette observation rejoint celle de Tialou et al. (2021) qui affirme que les cultures se pratiquant en milieu ouvert créent un milieu bien éclairé qui favorise le développement des espèces héliophiles.

Les populations des localités du Centre et de l'Est constituent le second groupe relativement important. Elles pratiquent beaucoup les cultures vivrières telles l'igname, et les légumes qui laissent souvent des espaces libres favorables à l'apparition de l'espèce Euphorbia heterophylla.

Les populations des localités de l'Ouest et du Sud constituent le groupe le moins important. Bien que pratiquant l'agriculture celles-ci sont plus tournés vers les cultures pérennes dont les couverts végétaux occupent presque la quasi-totalité des espaces cultivés, empêchant ainsi la germination des graines d'Euphorbia heterophylla. Cette faible proportion surtout au niveau du sud peut s'expliquer par la forte urbanisation et la migration de la population vers les villes, à la recherche d'emplois non agricoles.

En ce qui concerne le mode de préparation, l'expression et le broyage sont les plus pratiqués. Cela pourrait s'expliquer par le fait que ces deux modes de préparation n'engendrent pas de transformations majeures au niveau des métabolites secondaires contenus dans les organes de la plante. Ce résultat diffère de celui de Bene et al. (2016) qui ont montré que la décoction est la technique de préparation la plus employée dans le département de Transua en Côte d'Ivoire. Cette différence peut être liée à la typologie des plantes étudiées ainsi que leurs organes.

La voie cutanée était le mode d'administration le plus utilisé. Ce résultat corrobore celui de Bene et al. (2016) qui ont montré que la voie cutanée (35\%) était la plus utilisée parmi les voies d'administration enregistrées.

Les recettes à base de feuille uniquement étaient majoritaires. Ce résultat rejoint celui de Ouattara et al. (2021) qui ont montré que les feuilles $(41,86 \%)$ étaient les plus sollicitées. Les feuilles constituent la partie la plus importante de la plante herbacée. Elles sont donc susceptibles de renfermer le maximum de métabolite sécondaire responsables des effets thérapeutiques.

\section{Conclusion}

L'étude ethnobotanique menée dans différentes zones agro-écologiques de la Côte d'Ivoire a révélé que le niveau de connaissance de l'espèce végétale Euphorbia heterophylla est élevé en milieu rural pour les dégâts qu'elle cause aux cultures. Cette plante présente trois formes de feuilles : elliptique ou ovale, violon et lancéolée à bordures dentées ou non. Ses différentes vertus médicinales sont reconnues par une frange restreinte de cette population. Elle est utilisée dans le traitement de diverses maladies principalement le paludisme et les morsures de serpent en traitement curatif et en prévention pour l'accouchement facile. L'organe le plus utilisé est la feuille et les modes de préparations les plus employés sont l'expression et le broyage. Les voies d'administration dominantes des remèdes étaient les voies cutanée et orale. Cette étude constitue une ouverture pour d'autres recherches en vue de la valorisation de cette plante. Elle peut en outre servir de donnée à approfondir dans le domaine médicinal et enrichir la pharmacopée en Côte d'Ivoire. 


\section{CONFLITS D'INTERETS}

Les auteurs déclarent qu'ils n'ont aucun conflit d'intérêts.

\section{CONTRIBUTIONS DES AUTEURS}

JAD a fait la prospection pour identifier les variétés répandues dans les différentes zones géographiques, effectué les enquêtes, le traitement des donnés relevées et la rédaction du manuscrit. CYB a donné l'orientation des travaux à réaliser sur le terrain et contribué à la recherche documentaire. DNK a prodigué des conseils pour l'obtention de certaines données au niveau de l'enquête et contribué à la rédaction du manuscrit. NGZ par sa lecture il a contribué à l'amélioration et à la validation du manuscrit.

\section{REMERCIEMENTS}

Les auteurs remercient les chefs de villages des différentes localités visitées ainsi que les différentes personnes qui se sont prêtées au questionnaire. Ils remercient également les guides interprètes pour leur disponibilité et leur franche collaboration. Ils témoignent aussi leur reconnaissance à l'endroit des enseignants-chercheurs du département agriculture et ressources animales de l'INP-HB pour leurs conseils avisés.

\section{REFERENCES}

Bene K, Camara D, Fofie NBY, Kanga Y, Yapi AB, YAPO YC, Ambe SA, Zirihi GN. 2016. Etude ethnobotanique des plantes médicinales utilisées dans le département de Transua, District du Zanzan (Côte d'Ivoire). Journal of Animal \& Plants Sciences, 27: 4230-4250. DOI: http:/www.m.elewa.org.JAPS

Falodun A, Okunrobo LO, Uzoamaka N. 2006. Phytochemical screening and antiinflammatory evaluation of methanolic and aqueous extracts of Euphorbia heterophylla L. (Euphorbiaceae), African Journal of Biotechnology, 5: 529-531. DOI:10.4314/AJB.V5I6

Ipou IJ. 2005. Biologie et écologie d'Euphorbia heterophylla L.
(Euphorbiaceae) en culture cotonnière, au nord de la Côte d'Ivoire. Thèse de doctorat de l'Université de Cocody, Abidjan, Côte d'Ivoire. 167 p.

James WH, Zhenxiang X, Ricarda R, Jess AP, Ya Y, Brian LD, Paul EB, Charles CD. Kenneth JW. 2014. Evolutionary bursts in Euphorbia (Euphorbiaceae) are linked with photosynthetic pathway. International Journal of Organic Evolution, 68-12: 3485-3504. DOI: 10.1111/evo.12534

Kadiyala G, Kadali R, Bannikuppe SV, Gurunathan J. 2015. Protective effect of Euphorbia hirta and its components against snake venom induced lethality. Journal of Ethnopharmacology, 165:180190.

DOI : https://doi.org/10.1016/j.jep.2015.02.044

Kone MW, Atindehon KK, Tere H, Traore D. 2002. Quelques plantes médicinales utilisées en pédiatrie traditionnelle dans la Région de Ferkessédougou (Côte d'Ivoire). Actes du colloque international Centre Suisse du 27-29 août 2001, Editions Universitaires de Côte d'Ivoire BIOTERRE, Revue Internationale. Sciences de la vie et de la Terre. $\mathrm{N}^{\circ}$ spécial : 30-36

Kouakou ND, Gnantro CT, Kouadio BK, Cho EMA-K, Amissa AA, Nogbou EA, Jean FG : 2009. Essai préliminaire de production d'œufs des poules pondeuses (ISA Warren) enrichis en acides gras polyinsaturés oméga 3 avec les graines de Euphorbia heterophylla L. International Journal of Biological and Chemical Sciences, 9(4): 1902-1909. DOI : http://dx.doi.org/10.4314/ijbcs.v9i4.15

N'guessan K. 1995. Contribution à l'étude ethnobotanique en pays Krobou, République de Côte d'Ivoire. Thèse de Doctorat de $3^{\text {ème }}$ cycle, Université de Cocody-Abidjan (Côte d'Ivoire), $557 \mathrm{p}$.

OMS. 2004. Stratégie OMS de coopération avec la République de Côte d'Ivoire 2004-2007. OMS, Bureau Régional pour l'Afrique, Brazzaville (République du Congo), $39 \mathrm{p}$. 
Ouattara D. 2006. Contribution à l'inventaire de plantes médicinales significatives utilisées dans la Région de Divo (Sud forestier de la Côte d'Ivoire et à la diagnose du poivrier de Guinée : Xylopia aethiopica (Dunal) A.Rich (Annonaceae). Thèse de Doctorat, Université de Cocody- Abidjan (Côte d'Ivoire), $184 \mathrm{p}$.

Ouattara KE, Doga D, Orsot BAMB, Zirihi GN. 2021. Plantes médicinales utilisées dans le traitement des maladies microbiennes dans la région du Hambol, nord de la Côte d'Ivoire. Journal of Animal \& Plant Sciences, 47: 8412-8425. DOI :

https://doi.org/10.35759/JAnmPISci.v471.6

Oyedum UM. : Antibacterial and toxicological effects of leaf extracts of Eurphorbia heterophylla on some enteric bacteria. Global Journal of Pure and Applied Sciences, 23: 59-67. DOI: 10.4314/gjpas.v23i1.7.

Tialou OF, Yapi FA, Kla K, Boraud KNM. 2021. Adventices majeures des parcelles industrielles de canne à sucre des unités agricoles intégrées en Côte d'Ivoire. International Journal of Biological and
Chemical Sciences, 15: 594-608. DOI : https://dx.doi.org/10.4314/ijbcs.v15i2.17

Tra Bi FH : 1997. Utilisation des plantes, par l'homme, dans les forêts classées du Haut Sassandra et Scio en Côte d'Ivoire, Thèse de Doctorat de $3^{\text {ème }}$ cycle, Université de Cocody-Abidjan, $212 \mathrm{p}$.

Vangah MO. 1986. Contribution à la connaissance des plantes médicinales utilisées par les ethnies Akans de la région littorale de la Côte d'Ivoire. Thèse de Doctorat de $3^{\text {ème }}$ cycle, Université Nationale d'Abidjan (Côte d'Ivoire), 464 p.

Yapi AB : 2013. Inventaire des plantes de la famille des Asteraceae des marchés de la commune d'ABOBO (Abidjan, 'Côte d'Ivoire), Mémoire de Master II de botanique, UFR Biosciences, Université Félix Houphouët-Boigny, $50 \mathrm{p}$.

Zirihi GN. 1991. Contribution au recensement, à l'identification et la connaissance de quelques espèces végétales utilisées dans la médecine traditionnelle et la pharmacopée chez les Bétés du département d'Issia, Côte d'Ivoire. Thèse de Doctorat de $3^{\text {ème }}$ cycle, Université d'Abidjan, $253 \mathrm{p}$. 